\title{
Robust spin-orbit coupling induced semimetallic state in hyperkagome iridate $\mathrm{Li}_{3} \operatorname{Ir}_{3} \mathrm{O}_{8}$
}

\author{
T. Takayama, ${ }^{1,2}$ A. N. Yaresko, ${ }^{1}$ A. S. Gibbs,${ }^{3}$ K. Ishii $\odot,{ }^{4}$ D. Kukusta, ${ }^{1}$ and H. Takagi ${ }^{1,2,5}$ \\ ${ }^{1}$ Max Planck Institute for Solid State Research, Heisenbergstrasse 1, 70569 Stuttgart, Germany \\ ${ }^{2}$ Institute for Functional Matter and Quantum Technologies, University of Stuttgart, Pfaffenwaldring 57, 70550 Stuttgart, Germany \\ ${ }^{3}$ ISIS Neutron and Muon Source, STFC Rutherford Appleton Laboratory, Chilton, Didcot, Oxon OX11 OQX, United Kingdom \\ ${ }^{4}$ Synchrotron Radiation Research Center, National Institutes for Quantum and Radiological Science and Technology, \\ Sayo, Hyogo 679-5148, Japan \\ ${ }^{5}$ Department of Physics, University of Tokyo, 7-3-1 Hongo, Tokyo 113-0033, Japan
}

(Received 23 October 2019; revised 10 March 2020; accepted 6 July 2020; published 23 July 2020)

\begin{abstract}
A hyperkagome iridate $\mathrm{Li}_{3} \mathrm{Ir}_{3} \mathrm{O}_{8}$ is synthesized by an ion-exchange reaction from $\mathrm{Na}_{4} \mathrm{Ir}_{3} \mathrm{O}_{8}$. The transport, magnetic, and thermodynamic measurements suggest a metallic state. The electronic structure calculation shows that $\mathrm{Li}_{3} \mathrm{Ir}_{3} \mathrm{O}_{8}$ hosts a semimetallic electronic structure produced by a competition between the formation of a localized molecular orbital of Ir $d$ electrons on the $\operatorname{Ir}_{3}$ triangles and the strong spin-orbit coupling as in the sister compound $\mathrm{Na}_{3} \mathrm{Ir}_{3} \mathrm{O}_{8}$. The semimetallic state induced by spin-orbit coupling is quite robust against the moderate change of lattice distortion associated with the different ionic radii of $\mathrm{Li}^{+}$and $\mathrm{Na}^{+}$.
\end{abstract}

DOI: 10.1103/PhysRevMaterials.4.075002

\section{INTRODUCTION}

In heavy $5 d$ transition-metal-based compounds, the strong spin-orbit coupling $\lambda_{\text {SO }}$ of $5 d$ electrons, comparable even to the on-site Coulomb repulsion $U$, has been shown to give rise to unprecedented electronic phases and functions. Complex iridium oxides with tetravalent $\mathrm{Ir}^{4+}$ have been playing a central role in such explorations [1]. When the $\operatorname{Ir}^{4+}\left(5 d^{5}\right)$ ions are coordinated octahedrally by oxygen ions in such complex iridates, all of the five electrons are accommodated in the lower triply degenerate $t_{2 g}$ orbitals due to the large cubic crystal field $\left(t_{2 g}-e_{g}\right)$ splitting overwhelming the effect of Hund's coupling. The spin-orbit coupling of Ir $5 d$ electrons as large as half an $\mathrm{eV}$ entangles the spin and the orbital moments and splits the $t_{2 g}$ manifold into the lower fully filled $J_{\text {eff }}=3 / 2$ state and the upper half-filled $J_{\text {eff }}=1 / 2$ state. With a moderately strong $U$ of a few $\mathrm{eV}$, a metallic state with the half-filled $J_{\text {eff }}=1 / 2$ band is known to switch very often to a Mott insulator. Such $J_{\text {eff }}=1 / 2$ Mott insulators have been extensively studied as a platform for exotic magnetic ground states due to their bond-sensitive magnetic interactions originating from the spin-orbital entanglement of $J_{\text {eff }}=1 / 2$ wave functions [2].

Among such $J_{\text {eff }}=1 / 2$ Mott insulators with octahedrally coordinated $\mathrm{Ir}^{4+}$ ions, hyperkagome iridate $\mathrm{Na}_{4} \mathrm{Ir}_{3} \mathrm{O}_{8}$ is the first material where an unconventional magnetic ground state was identified [3]. The crystal structure of $\mathrm{Na}_{4} \mathrm{Ir}_{3} \mathrm{O}_{8}$ consists of an edge-sharing network of $\mathrm{IrO}_{6}$ octahedra, where $\mathrm{Ir}^{4+}$ ions occupy $3 / 4$ of the B-site pyrochlore sublattice in the

Published by the American Physical Society under the terms of the Creative Commons Attribution 4.0 International license. Further distribution of this work must maintain attribution to the author(s) and the published article's title, journal citation, and DOI. Open access publication funded by the Max Planck Society. spinel-like structure, and the remaining $1 / 4$ of $\mathrm{B}$ sites are filled by $\mathrm{Na}^{+}$ions. The Ir and $\mathrm{Na}$ atoms with a 3:1 ratio adopt an ordered arrangement in the pyrochlore lattice, forming triangles of Ir atoms connected by their corners in a threedimensional lattice dubbed the hyperkagome lattice. As is in the spinel-type structure, the $\mathrm{Ir}^{4+}$ ions on the hyperkagome sublattice are connected to their neighbors by sharing the edges of the $\mathrm{IrO}_{6}$ octahedron. The rest of $\mathrm{Na}$ atoms fill the other octahedral voids, rather than the tetrahedral A site in contrast to the case of spinels. The hyperkagome lattice renders a strong geometrical frustration when the magnetic moments of $\mathrm{Ir}^{4+}$ ions couple antiferromagnetically. Indeed, $\mathrm{Na}_{4} \mathrm{Ir}_{3} \mathrm{O}_{8}$ was reported to show no magnetic ordering down to $2 \mathrm{~K}$ despite the strong antiferromagnetic interactions inferred from the negative Curie-Weiss temperature $\theta_{\mathrm{CW}}$ of $\sim-650 \mathrm{~K}$. It was therefore proposed as the first candidate for a threedimensional quantum spin liquid. Recent nuclear magnetic resonance and muon spin relaxation studies point to the appearance of a spin-frozen state or quasistatic order with slow fluctuations, potentially associated with the disordered arrangement of $\mathrm{Na}$ atoms in the octahedral voids [4,5].

$\mathrm{Na}_{4} \mathrm{Ir}_{3} \mathrm{O}_{8}$ had in fact been discovered even before the significance of spin-orbit coupling of $5 d$ electrons has been widely recognized. Following the discovery of $\mathrm{Na}_{4} \mathrm{Ir}_{3} \mathrm{O}_{8}$, the crucial role of spin-orbit coupling in the electronic and magnetic properties has been theoretically discussed [6-8]. In particular, as the $\mathrm{IrO}_{6}$ octahedra in $\mathrm{Na}_{4} \mathrm{Ir}_{3} \mathrm{O}_{8}$ are connected by their edges and form $90^{\circ} \mathrm{Ir}-\mathrm{O}_{2}-\mathrm{Ir}$ planar bonds, Kitaev-type bond-dependent Ising coupling, and other anisotropic interactions should be present in addition to the isotropic Heisenberg coupling as discussed in the honeycomb-based iridates [6,911]. The negative $\theta_{\mathrm{CW}}$ of $\sim-650 \mathrm{~K}$ is observed in the high temperature Curie-like magnetic susceptibility, implying that the antiferromagnetic Heisenberg interaction is dominant over the ferromagnetic Kitaev coupling. The magnitude of the antiferromagneic $\theta_{\mathrm{CW}}$ is much larger in $\mathrm{Na}_{4} \mathrm{Ir}_{3} \mathrm{O}_{8}$ than in the 
(a)

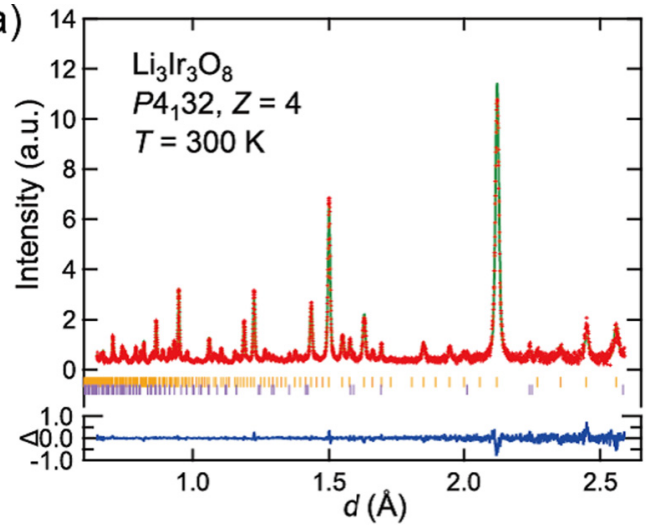

(b)

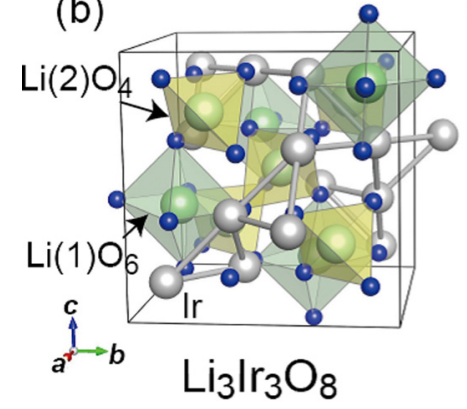

(c)

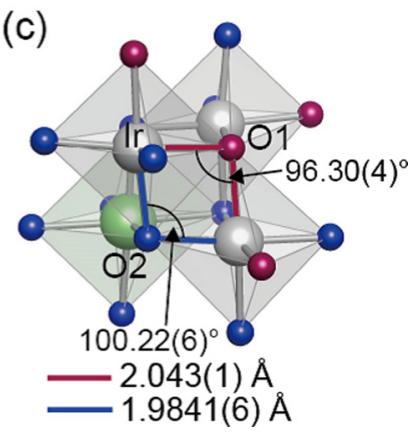

FIG. 1. (a) Neutron diffraction pattern of $\mathrm{Li}_{3} \mathrm{Ir}_{3} \mathrm{O}_{8}$ (backscattering bank, HRPD). The red dots and green line represent the observed pattern and the Rietveld fit profile, respectively. The orange and purple bars indicate the reflection positions of $\mathrm{Li}_{3} \mathrm{Ir}_{3} \mathrm{O}_{8}$ and $\mathrm{IrO}_{2}$ as an impurity phase, respectively. The refined amount of $\mathrm{IrO}_{2}$ was $\sim 4$ wt.\%. The blue line shows the difference between the observed and calculated profiles $\Delta$. (b) Crystal structure of $\mathrm{Li}_{3} \mathrm{Ir}_{3} \mathrm{O}_{8}$. The gray, light green, and blue spheres represent $\mathrm{Ir}, \mathrm{Li}$, and $\mathrm{O}$ atoms, respectively. The Ir atoms form a network of corner-shared triangles (hyperkagome lattice). (c) Local structure around $\mathrm{IrO}_{6}$ octahedra. To highlight the two oxygen sites, $\mathrm{O} 1$ and $\mathrm{O} 2$ are shown in different colors. The edge-shared Ir-O2-Ir plaquette is asymmetrically distorted because of distinct Ir-O bond lengths. The crystal structures are visualized using VESTA software [30].

honeycomb-based iridates. The enhanced antiferromagnetic interaction and possibly reduced anisotropic ferromagnetic coupling may be related to the strong noncubic distortion of $\mathrm{IrO}_{6}$ octahedra in $\mathrm{Na}_{4} \mathrm{Ir}_{3} \mathrm{O}_{8}$ with two distinct Ir-O bond lengths in the $\mathrm{Ir}^{-} \mathrm{O}_{2}$-Ir edge-shared bond [see Fig. 1(c)].

During the optimization of the synthetic process of $\mathrm{Na}_{4} \mathrm{Ir}_{3} \mathrm{O}_{8}$, a closely related compound $\mathrm{Na}_{3} \mathrm{Ir}_{3} \mathrm{O}_{8}$ with the same hyperkagome network of $\mathrm{IrO}_{6}$ octahedra was discovered. $\mathrm{Na}_{3} \mathrm{Ir}_{3} \mathrm{O}_{8}$ may be viewed as a $1 / 3$ hole-doped analog of the $J_{\text {eff }}=1 / 2$ Mott insulator $\mathrm{Na}_{4} \mathrm{Ir}_{3} \mathrm{O}_{8}$ and shows metallic behavior [12]. $\mathrm{Na}_{3} \mathrm{Ir}_{3} \mathrm{O}_{8}$ was found to be a semimetal rather than having a correlated metallic state with a large Fermi surface, which would be naively expected for a doped Mott insulator. The semimetallic state was shown to be produced by a subtle competition between the band gap produced by the formation of molecular orbital on the $\mathrm{Ir}_{3}$ triangles and the suppression of the gap by the formation of a $J_{\text {eff }}=1 / 2$-like state due to the strong spin-orbit coupling.

We note here that the $\mathrm{IrO}_{6}$ octahedron in $\mathrm{Na}_{3} \mathrm{Ir}_{3} \mathrm{O}_{8}$ is heavily distorted, which apparently influences the formation of the molecular orbital state and also the $J_{\text {eff }}=1 / 2$ state, through the noncubic crystal field. The distortion of $\mathrm{IrO}_{6}$ is likely associated with the large difference of ionic radius between $\mathrm{Na}^{+}$and $\mathrm{Ir}^{4+}$ ions that share the pyrochlore lattice. By changing such a distortion, the competition of molecularorbital formation and $J_{\text {eff }}=1 / 2$ formation should be modified and different magnetic and electronic ground states might emerge. One of the possible approaches to change the degree of distortion of $\mathrm{IrO}_{6}$ octahedra is to replace the $\mathrm{Na}$ ions with monovalent ions with a different radius such as $\mathrm{Li}$. We were therefore motivated to synthesize $\mathrm{Li}_{3} \mathrm{Ir}_{3} \mathrm{O}_{8}$ to chemically control the competition of the two factors. However, standard solid-state reaction has been unsuccessful so far in synthesizing $\mathrm{Li}_{3} \mathrm{Ir}_{3} \mathrm{O}_{8}$. By employing an ion-exchange technique instead, we have successfully obtained a hyperkagome iridate $\mathrm{Li}_{3} \mathrm{Ir}_{3} \mathrm{O}_{8} . \mathrm{Li}_{3} \mathrm{Ir}_{3} \mathrm{O}_{8}$ was found to host a semimetallic electronic structure induced by spin-orbit coupling as in $\mathrm{Na}_{3} \mathrm{Ir}_{3} \mathrm{O}_{8}$, suggesting the robustness of the semimetallic state to the modification of lattice distortions.

\section{EXPERIMENTAL AND CALCULATION METHODS}

The polycrystalline sample of $\mathrm{Li}_{3} \mathrm{Ir}_{3} \mathrm{O}_{8}$ was synthesized by an ion-exchange reaction. The powder sample of $\mathrm{Na}_{4} \mathrm{Ir}_{3} \mathrm{O}_{8}$ was obtained by a solid-state reaction between $\mathrm{Na}_{2} \mathrm{CO}_{3}$ and $\mathrm{IrO}_{2}$ as a precursor. The precursor was confirmed to be $\mathrm{Na}_{4} \mathrm{Ir}_{3} \mathrm{O}_{8}$, not $\mathrm{Na}_{3} \operatorname{Ir}_{3} \mathrm{O}_{8}$, by magnetization measurements. The ion-exchange was performed by mixing the precursor powder with $\mathrm{LiNO}_{3}$ of 40 times excess amount in molar ratio. The mixture was heated at $400^{\circ} \mathrm{C}$ for $50 \mathrm{~h}$ in air. The obtained product was rinsed with distilled water to remove residual $\mathrm{LiNO}_{3}$ and $\mathrm{NaNO}_{3}$ byproduct. The product is stable in air.

The structural analysis of the product with $\mathrm{x}$-ray diffraction was not easy because of much smaller scattering factors of $\mathrm{Li}$ and $\mathrm{O}$ compared with that of Ir. We thus performed neutron diffraction, which is intrinsically advantageous for the determination of positions of light elements. Since ${ }^{191} \mathrm{Ir}$, a substantial component of the natural isotopic mixture, is known to suffer significantly from the absorption of neutrons, we synthesized an isotope-enriched $\mathrm{Li}_{3}^{193} \operatorname{Ir}_{3} \mathrm{O}_{8}$ in which the neutron absorption can be suppressed by a factor of 4 as compared with the sample without isotopic enrichment [13]. The neutron diffraction experiment was conducted at room temperature, with the sample contained in a thin-walled vanadium foil can, on High Resolution Powder Diffractometer (HRPD) at the ISIS Neutron and Muon Source. We used the GSAS software for the Rietveld refinement of the neutron diffraction pattern $[14,15]$.

Magnetization, transport and thermodynamic properties have been measured with commercial apparatus (Quantum Design MPMS and PPMS). A cold-pressed pellet was used for those measurements. The Seebeck coefficient was measured using a home-built system.

Resonant inelastic $x$-ray scattering (RIXS) measurement at the $\operatorname{Ir} L_{3}$ edge was performed on a polycrystalline pellet. The RIXS experiment was carried out at BL11XU of SPring8 . The incident $\mathrm{x}$-ray was monochromized by $\mathrm{Si}(111)$ double monochromator and the secondary $\mathrm{Si}(844)$ four-crystal monochromator. The energy of incident $\mathrm{x}$-ray was tuned to $11.215 \mathrm{keV}$. The scattering angle $(2 \theta)$ was kept at $90^{\circ}$, and 
TABLE I. Refined structural parameters for $\mathrm{Li}_{3} \mathrm{Ir}_{3} \mathrm{O}_{8}$ at room temperature. The space group is $P 4_{1} 32$ (No. 213) and $Z=4$. The lattice constant is $a=8.48614(18) \AA$. $g$ and $U_{\text {iso }}$ denote site occupancy and the isotropic displacement parameter, respectively. $U_{\text {iso }}$ was constrained to be equal across the Li sites during the refinement. The refinement indices are $R_{p}=5.12 \%, R_{w p}=3.28 \%$, and $\chi^{2}=$ 2.847 [19].

\begin{tabular}{lcccccl}
\hline \hline Atom & Site & $g$ & $x$ & $y$ & $z$ & \multicolumn{1}{c}{$U_{\text {iso }}\left(\AA^{2}\right)$} \\
\hline Li1 & $4 b$ & 1 & $1 / 8$ & $1 / 8$ & $1 / 8$ & $0.0158(4)$ \\
Li2 & $8 c$ & 1 & $0.2468(3)$ & $x$ & $x$ & $0.0158(4)$ \\
Ir1 & $12 d$ & 1 & $0.63212(6)$ & $x+1 / 4$ & $5 / 8$ & $0.00563(11)$ \\
O1 & $8 c$ & 1 & $0.10783(10)$ & $x$ & $x$ & $0.0053(3)$ \\
O2 & $24 e$ & 1 & $0.12446(8)$ & $0.89195(6)$ & $0.88544(11)$ & $0.00711(12)$ \\
\hline
\end{tabular}

the incident $\mathrm{x}$-rays were $\pi$-polarized to minimize the elastic scattering. The scattered $x$-rays were analyzed by a diced and spherically-bent $\mathrm{Si}(844)$ analyzer and collected by Mythen microstrip x-ray detector (Dectris). The measurement was performed at $10 \mathrm{~K}$. The total energy resolution was about $50 \mathrm{meV}$

The electronic structure calculation was performed based on the local density approximation with using the fully relativistic linear muffin-tin orbital (LMTO) method implemented in the PY LMTO code [16]. Spin-orbit coupling was incorporated by solving the four-component Dirac equation inside an atomic sphere. The effect of spin-orbit coupling is incorporated by calculating the $J$-resolved density of states where $J=l \pm 1 / 2$ is the total angular momentum for the $5 d$ states. The RIXS spectra were calculated based on the single-particle fully relativistic electronic band structure and performed in the dipolar approximation with the use of experimental geometry $[17,18]$.

\section{RESULT AND DISCUSSION}

\section{A. Structural analysis}

The powder $\mathrm{x}$-ray diffraction showed that the product consists of almost single phase except for a small amount of impurity phase of $\mathrm{IrO}_{2}(\sim 4 \mathrm{wt} . \%)$. The diffraction pattern of the product is similar to that of the parent $\mathrm{Na}_{4} \mathrm{Ir}_{3} \mathrm{O}_{8}$ yet with a substantially reduced lattice spacing (Supplementary Fig. S1 [19]). The smaller lattice constant is consistent with the replacement of $\mathrm{Na}$ with $\mathrm{Li}$ atoms. The energy-dispersive $\mathrm{x}$-ray spectrum did not find any trace of $\mathrm{Na}$ ions in the product within the given resolution (Supplemental Material Fig. S2 [19]). These results imply the successful substitution of $\mathrm{Na}$ atoms with $\mathrm{Li}$. The powder neutron diffraction pattern at room temperature on the ${ }^{193} \mathrm{Ir}$ isotope-enriched sample is shown in Fig. 1(a). The significant difference of neutron scattering lengths between $\mathrm{Li}$ and $\mathrm{Na}$ enables a reliable refinement of their positions and occupancies even if $\mathrm{Na}$ atoms remain partially. The Rietveld analysis showed that the structural model of $\mathrm{Li}_{3} \mathrm{Ir}_{3} \mathrm{O}_{8}$ gives the best fitting result amongst the models employed (see Supplemental Material [19]). The results of the Rietveld refinement are listed in Table I.

The refined structure of $\mathrm{Li}_{3} \mathrm{Ir}_{3} \mathrm{O}_{8}$ is identical to that of $\mathrm{Na}_{3} \mathrm{Ir}_{3} \mathrm{O}_{8}$ [12]. The Li2 atom is coordinated tetrahedrally with

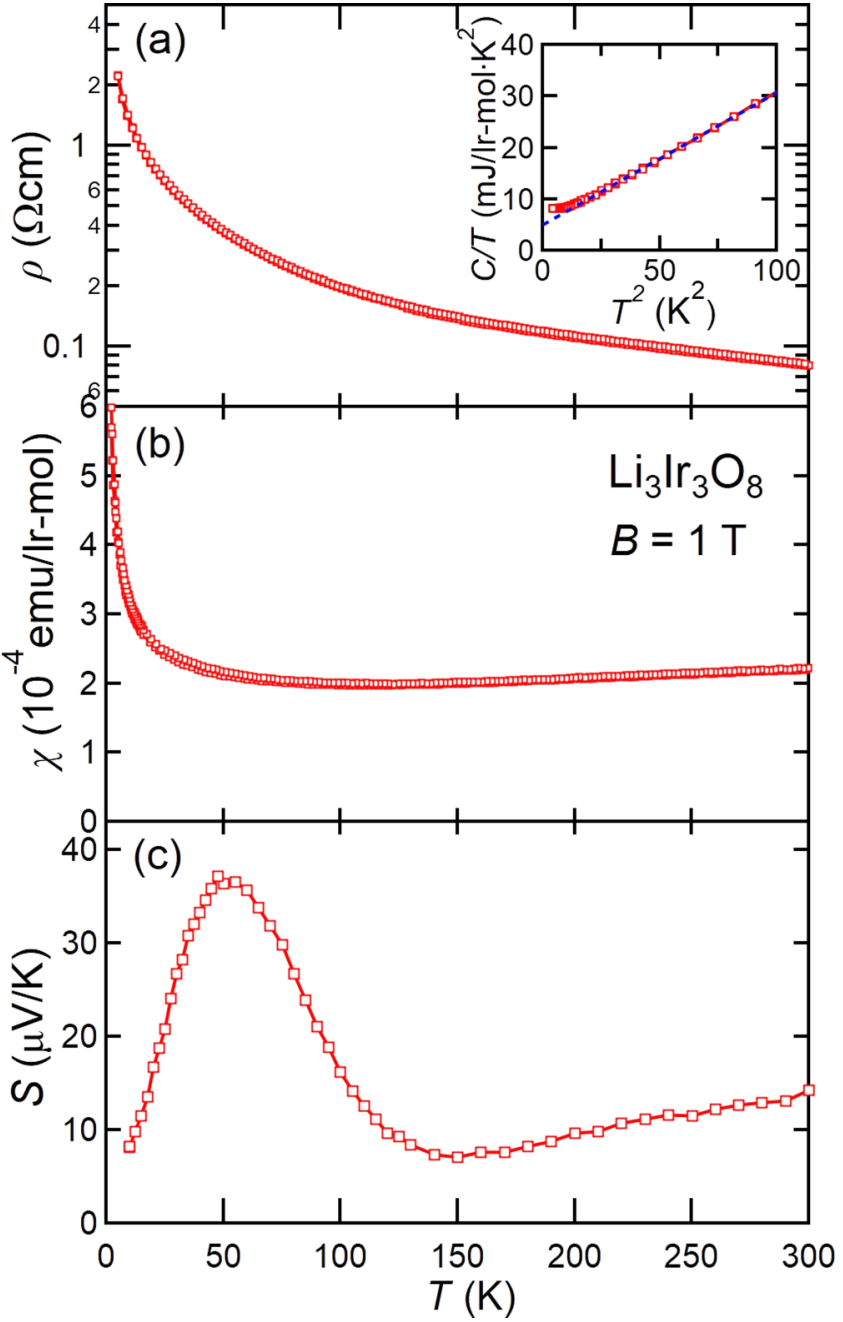

FIG. 2. Transport and magnetic properties of $\mathrm{Li}_{3} \mathrm{Ir}_{3} \mathrm{O}_{8}$ polycrystalline sample displaying (a) resistivity $\rho(T)$, (b) magnetic susceptibility $\chi(T)$ measured at $1 \mathrm{~T}$, and (c) Seebeck coefficient $S(T)$, respectively. The inset of (a) shows a specific heat divided by temperature, $C(T) / T$, as a function of squared temperature.

oxygen atoms, whereas the $\mathrm{Li} 1$ atoms form $\mathrm{LiO}_{6}$ octahedra sharing their edges with $\mathrm{IrO}_{6}$ octahedra in the hyperkagome lattice [Fig. 1(b)]. While all the $\mathrm{Na}$ ions occupy octahedral sites in the precursor $\mathrm{Na}_{4} \mathrm{Ir}_{3} \mathrm{O}_{8}, 2 / 3$ of $\mathrm{Li}$ atoms occupy the tetrahedral site in $\mathrm{Li}_{3} \mathrm{Ir}_{3} \mathrm{O}_{8}$ likely because the smaller $\mathrm{Li}$ ions preferentially enter the tetrahedral site with shorter Li-O length. $\mathrm{Li}_{3} \mathrm{Ir}_{3} \mathrm{O}_{8}$ can therefore be viewed as an ordered spinel structure $2 \times \mathrm{Li}\left(\mathrm{Li}_{1 / 4} \mathrm{Ir}_{3 / 4}\right)_{2} \mathrm{O}_{4}$ in which the B-site pyrochlore lattice of the spinel is occupied by $1 / 4$ of $\mathrm{Li}$ and $3 / 4$ of Ir atoms comprising the hyperkagome lattice. The $\mathrm{Li}$ atoms are fully ordered without any fractional occupations in contrast to the $\mathrm{Na}$ atoms in $\mathrm{Na}_{4} \mathrm{Ir}_{3} \mathrm{O}_{8}$.

\section{B. Transport and magnetic properties of $\mathrm{Li}_{3} \mathrm{Ir}_{3} \mathrm{O}_{8}$}

The chemical formula $\mathrm{Li}_{3} \mathrm{Ir}_{3} \mathrm{O}_{8}$ indicates that Ir ions have a noninteger valence state of $\mathrm{Ir}^{4.33+}$, which would naively be expected to lead to a metallic state. The resistivity $\rho(T)$ of $\mathrm{Li}_{3} \mathrm{Ir}_{3} \mathrm{O}_{8}$ measured with a cold-pressed pellet displays a seemingly semiconducting behavior [Fig. 2(a)]. However, it shows 
only a factor of 20 increase between 300 and $5 \mathrm{~K}$, which would be too weak to be a gapped semiconductor, and does not follow an activation behavior, although the magnitude of resistivity is as high as $100 \mathrm{~m} \Omega \mathrm{cm}$ at room temperature. The measured resistance may be dominated by grain boundary resistance as the pellet was made without any sintering, or possibly affected by disorder due to small nonstoichiometry [19]. The intrinsic resistivity can be orders of magnitude lower and possibly metallic. We note that an insulating behavior of resistivity, despite intrinsic metallic conduction, has been observed in the polycrystalline samples of transition-metal oxides, for example $\mathrm{LiV}_{2} \mathrm{O}_{4}$ [22].

In order to confirm this point, the Seebeck coefficient $S(T)$, which is known to be insensitive to the grain boundary effect, was measured on the pellet. $S(T)$ is found to be positive value at room temperature and decreases upon cooling as in a metal. This provides a support for a metallic ground state since $S(T)$ of a semiconductor would increase on cooling due to the reduction of thermally excited carriers. When the temperature is lowered further, $S(T)$ increases steeply below $120 \mathrm{~K}$, shows a broad peak at around $60 \mathrm{~K}$, and decreases at low temperatures. As no signature of a phase transition was evident at this temperature in magnetic susceptibility $\chi(T)$ or specific heat $C(T)$, this broad peak in $S(T)$ is likely attributed to a phonondrag effect. The phonon-drag effect is often seen in systems with small carrier numbers such as narrow-gap semiconductors or semimetals with strong electron-phonon coupling [23].

Further support for a metallic ground state is obtained from the low temperature specific heat $C(T)$ shown in the inset of Fig. 2(a), plotted as $C(T) / T$ as a function of $T^{2}$, and the magnetic susceptibility $\chi(T)$ in Fig. 2(b). The extrapolation of $C(T) / T$ towards $0 \mathrm{~K}$, namely the electronic specific heat coefficient $\gamma$ shows a finite value of $\sim 5.0 \mathrm{~mJ} / \mathrm{Ir} \mathrm{mol} \mathrm{K}{ }^{2}$, which cannot be accounted for by the presence of $\mathrm{IrO}_{2}$ impurity [24]. This indicates a finite density of states at the Fermi energy $E_{\mathrm{F}}$. The magnitude of $\gamma$ is only a factor of 2 larger than that obtained from the band calculation shown below which indicates a semimetallic band structure $\left(\gamma_{\text {calc }} \sim 2.2 \mathrm{~mJ} / \mathrm{Ir} \mathrm{mol} \mathrm{K}{ }^{2}\right)$. The magnetic susceptibility $\chi(T)$ is paramagnetic and almost temperature-independent. It decreases only slightly on cooling from room temperature, followed by the low-temperature Curie-like upturn. This upturn is suppressed by increasing magnetic fields and thus likely due to defects or minor impurities, which also contribute to $C(T)$ at low temperatures [19]. This excludes the presence of localized magnetic moments and hence a Mott insulating ground state. The intrinsic susceptibility at low temperatures $\chi_{0}$ is estimated to be $\sim 1.9 \times 10^{-4}$ $\mathrm{emu} / \mathrm{Ir}$ mol. Note that this value likely contains the contribution from Van Vleck paramagnetic susceptibility which can be of the order of $10^{-4} \mathrm{emu} / \mathrm{Ir}$ mol [25]. Thus, the Pauli paramagnetic susceptibility would be even smaller. All those results suggest that $\mathrm{Li}_{3} \mathrm{Ir}_{3} \mathrm{O}_{8}$ is a paramagnetic metal with a moderate density of states at the Fermi energy. The values of $\gamma$ and $\chi_{0}$ are close to those of the sister compound $\mathrm{Na}_{3} \mathrm{Ir}_{3} \mathrm{O}_{8}$ $[12,19]$, which may point to the similar electronic structure.

\section{Electronic structure of $\mathrm{Li}_{3} \mathrm{Ir}_{3} \mathrm{O}_{8}$}

In order to obtain insights into the electronic structure, we carried out Ir $L_{3}$-edge RIXS measurements and

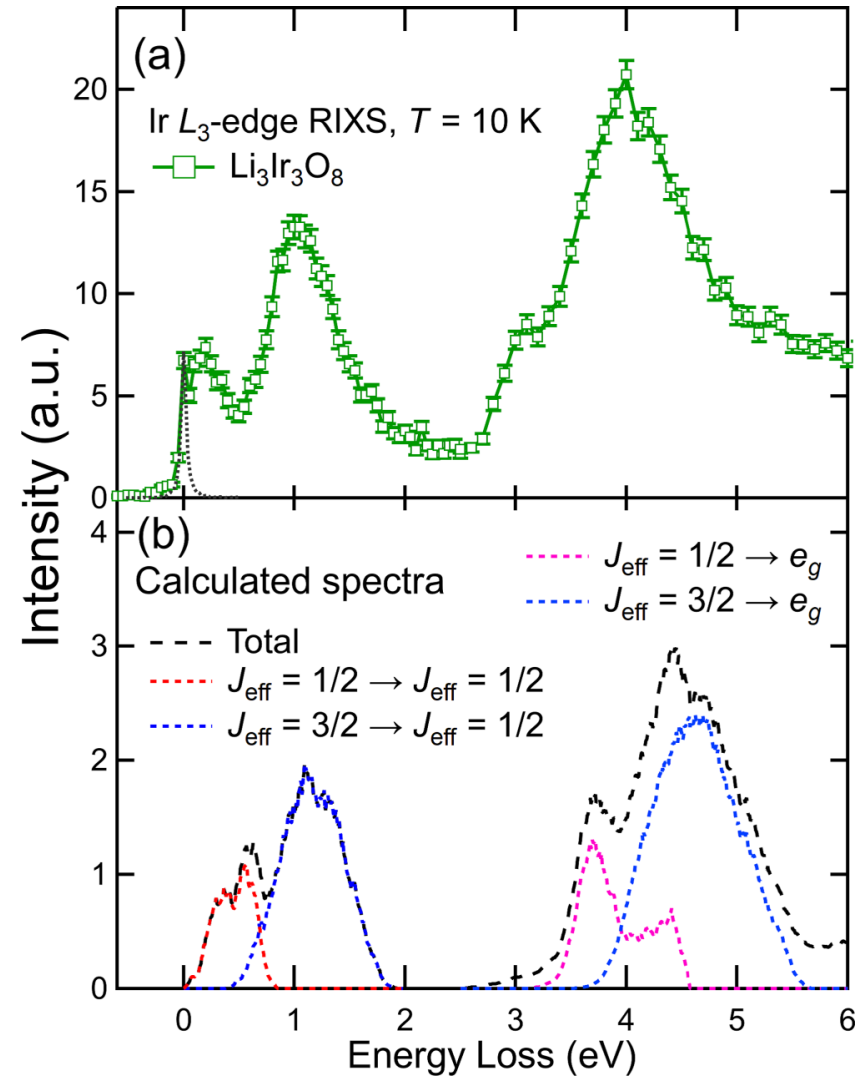

FIG. 3. (a) Ir $L_{3}$-edge RIXS spectrum of $\mathrm{Li}_{3} \mathrm{Ir}_{3} \mathrm{O}_{8}$ at $10 \mathrm{~K}$. The dotted line shows the elastic signal with normalized intensity, separately measured with the $\sigma$-polarized incident x rays. (b) The calculated RIXS spectrum resolved into each $d-d$ excitation based on the band structure calculation shown in Fig. 4(c).

electronic structure calculations on $\mathrm{Li}_{3} \mathrm{Ir}_{3} \mathrm{O}_{8}$. The obtained RIXS spectrum is displayed in Fig. 3(a). In addition to the elastic scattering, we find three features in the energy range below $6 \mathrm{eV}$. The broad peak centered at $4.0 \mathrm{eV}$ corresponds to the excitation from $t_{2 g}$ to $e_{g}(10 \mathrm{Dq})$. This value is slightly larger than that of $\mathrm{Na}_{4} \mathrm{Ir}_{3} \mathrm{O}_{8}(\sim 3.8 \mathrm{eV})$, but comparable to that of $\mathrm{Na}_{3} \mathrm{Ir}_{3} \mathrm{O}_{8}$ [12]. The larger split of $t_{2 g}-e_{g}$ levels is ascribed to the shorter Ir-O bond lengths due to the higher iridium valence of $4.33+$. The peaks below $2 \mathrm{eV}$ represent the excitations within the $t_{2 g}$ manifold, where the two peaks at $\sim 0.3$ and $\sim 1.1 \mathrm{eV}$ are observed. Obviously, the two peak structure is quite different from those of canonical $J_{\text {eff }}=1 / 2$ Mott insulators like $\mathrm{Sr}_{2} \mathrm{IrO}_{4}$ and $\mathrm{Na}_{2} \mathrm{IrO}_{3}$, where a relatively sharp excitation between the fully occupied $J_{\text {eff }}=3 / 2$ state and the empty $J_{\text {eff }}=1 / 2$ state is seen around $0.7 \mathrm{eV}$ corresponding to $3 / 2 \lambda_{\text {so }}[26,27]$. If the system were a metal with a single $J_{\text {eff }}=$ 1/2-band doped with $1 / 3$ hole, a single peak corresponding to the excitation from the fully occupied $J_{\text {eff }}=3 / 2$ band to the empty $J_{\text {eff }}=1 / 2$ band with $1 / 3$ hole doping would be observed. The presence of two peaks means that the actual band structure should be more complicated than the single and doped $J_{\text {eff }}=1 / 2$ band.

The electronic structure calculations indeed show that $\mathrm{Li}_{3} \mathrm{Ir}_{3} \mathrm{O}_{8}$ is a band insulator when spin-orbit coupling is neglected as depicted in Figs. 4(a) and 4(b). The origin of 

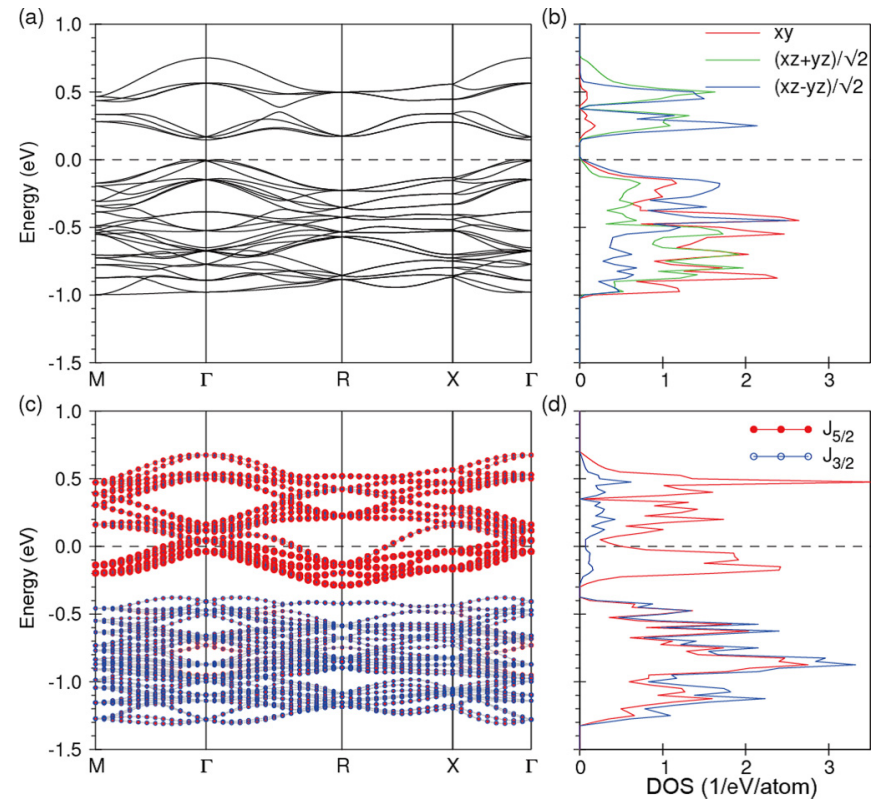

FIG. 4. Electronic structure of $\mathrm{Li}_{3} \mathrm{Ir}_{3} \mathrm{O}_{8}$. (a), (b) band structure and density of states for Ir $5 d$ state calculated without spin-orbit coupling. The $d_{x y}$ orbital ( $\left.x y\right)$ is fully occupied, while the other two $t_{2 g}$ orbitals form bonding and antibonding molecular orbitals [19]. (c), (d) band structure and density of state for Ir $5 d$ states calculated with spin-orbit coupling included. The density of states is resolved into total angular momentum of $5 d$ states with $J_{5 / 2}$ and $J_{3 / 2}$ wave function. The size of red(blue) circles in (c) represents the weights of $d$-orbital characters with $J_{5 / 2}\left(J_{3 / 2}\right)$, respectively.

the band insulating state can be understood as a formation of molecular orbitals of $\mathrm{Ir} d$ orbitals on the $\mathrm{Ir}_{3}$ triangles, an essential structural ingredient of the hyperkagome lattice as in the case of $\mathrm{Na}_{3} \mathrm{Ir}_{3} \mathrm{O}_{8}$ (see Supplemental Material [19] for the detailed description of molecular orbital formation). In the absence of spin-orbit coupling, one of the $t_{2 g}$ orbitals $\left(d_{x y}\right)$ has a lower energy because of distinct bond-lengths between Ir-O1 and Ir-O2 and is fully filled [Fig. 4(b)]. Note that we take a local-axis frame in which the $x y$ plane corresponds to the one containing two $\mathrm{O} 1$ atoms (see Supplemental Material Fig. S5). The other two $t_{2 g}$ orbitals hybridize with their equivalent orbital of neighboring Ir ions through Ir $5 d-\mathrm{O} 2$ $2 p$-Ir $5 d$ hybridization. The electron hopping path through the hybridization is confined within one of the $\mathrm{Ir}_{3}$ triangles in the hyperkagome lattice, giving rise to the formation of localized molecular orbitals on each $\mathrm{Ir}_{3}$ triangle. Each $\mathrm{Ir}_{3}$ triangle unit accommodates four bonding and two antibonding molecular orbitals, and three $d_{x y}$ orbitals. As each $\mathrm{Ir}^{4.33+}$ ion has $4.67 d$ electrons, there are $14 d$ electrons in an $\operatorname{Ir}_{3}$ molecule. The three $d_{x y}$ and four bonding molecular orbitals are fully filled with $14 d$ electrons and a gap exists between the bonding and the antibonding molecular orbitals. Since the molecular orbitals on $\mathrm{Ir}_{3}$ triangle are in a first approximation orthogonal to each other, all of the electrons in the molecular orbitals are almost localized within the $\operatorname{Ir}_{3}$ molecule, giving rise to a band insulator with a narrow band width and a bonding-antibonding gap. However, such a band insulating state is incompatible with a finite density of states at the Fermi level observed experimentally.
The spin-orbit coupling of Ir in reality reconstructs the electronic structure substantially. The Kramers degeneracy of bands is lifted except for the time-reversal-invariant momenta because of the anti-symmetric spin-orbit coupling in a noncentrosymmetric crystal structure [Fig. 4(c)]. For an isolated regular $\mathrm{IrO}_{6}$ octahedron, the $t_{2 g}$ manifold splits into a lower $J_{\text {eff }}=3 / 2$ state with $J_{5 / 2}$ and $J_{3 / 2}$ characters and upper $J_{\text {eff }}=1 / 2$ manifold with a purely $J_{5 / 2}$ wave function. In $\mathrm{Li}_{3} \mathrm{Ir}_{3} \mathrm{O}_{8}$, the states between -1.3 and $-0.4 \mathrm{eV}$ can be assigned to the $J_{\text {eff }}=3 / 2$ state whereas the ones between -0.3 and $-0.6 \mathrm{eV}$ have primarily $J_{\text {eff }}=1 / 2$ character as depicted in Fig. 4(d). There is a non-negligible $J_{3 / 2}$ component in the upper manifold, likely induced by the distortion of $\mathrm{IrO}_{6}$ and intersite orbital mixing. Inheriting the band gap of the nonrelativistic band structure due to the formation of molecular orbitals, the $J_{\text {eff }}=1 / 2$-like bands split into two groups which marginally overlap at $E_{\mathrm{F}}$, making the system semimetallic. The hole pockets are located around $\Gamma$ point, while electron pockets reside at the $R$ point. The hole bands around the $\Gamma$ point are less dispersive than the electron bands.

The RIXS spectrum of $\mathrm{Li}_{3} \mathrm{Ir}_{3} \mathrm{O}_{8}$ can be well accounted for by the band structure including spin-orbit coupling. The calculated spectrum based on the electronic structure of Fig. 4(c) is displayed in Fig. 3(b). The peak at $\sim 1.2 \mathrm{eV}$ can be understood as the excitation from the $J_{\text {eff }}=3 / 2$ bands to empty $J_{\text {eff }}=1 / 2$ bands above $E_{\mathrm{F}}$. The low-energy peak at around $0.3 \mathrm{eV}$ is the excitation between the $J_{\text {eff }}=1 / 2$ valence bands and conduction bands. The agreement of lowenergy excitations between the calculated and experimental RIXS spectra evidences the validity of calculated electronic structure, namely a spin-orbit induced semimetallic state in $\mathrm{Li}_{3} \mathrm{Ir}_{3} \mathrm{O}_{8}$. The obtained RIXS spectrum of $\mathrm{Li}_{3} \mathrm{Ir}_{3} \mathrm{O}_{8}$ is almost identical with that of $\mathrm{Na}_{3} \mathrm{Ir}_{3} \mathrm{O}_{8}$ [19], which also supports the similar semimetallic electronic structure.

The energy of $t_{2 g}-e_{g}$ excitation centered at around $4.0 \mathrm{eV}$ is smaller than the calculated result $(\sim 4.3 \mathrm{eV})$. As we observed a similar RIXS spectrum in $\mathrm{Na}_{3} \mathrm{Ir}_{3} \mathrm{O}_{8}$ [19], this is too large to be ascribed to the possible non-stoichiometry of the sample, namely a small deviation of valence state from $\mathrm{Ir}^{4.33+}$. The discrepancy may be caused by the spherically averaged potential and/or basis set used for the electronic structure calculation as the position of the $\operatorname{Ir} e_{g}$ state is quite sensitive to the choice of basis set.

\section{Comparison with $\mathrm{Na}_{3} \mathrm{Ir}_{3} \mathrm{O}_{8}$}

The hyperkagome iridate $\mathrm{Li}_{3} \mathrm{Ir}_{3} \mathrm{O}_{8}$ crystallizes in an ordered spinel structure as in the sister compound $\mathrm{Na}_{3} \mathrm{Ir}_{3} \mathrm{O}_{8}$. The ionic-size mismatch between $\mathrm{Ir}^{4+}$ and $\mathrm{Li}^{+}$ions, which share the B-site pyrochlore lattice of spinel, is smaller than that in $\mathrm{Na}_{3} \mathrm{Ir}_{3} \mathrm{O}_{8}$ [28]. This moderately reduces the distortion of $\mathrm{IrO}_{6}$ octahedra; the $\mathrm{Ir}-\mathrm{O} 1(\mathrm{O} 2)$ - $\mathrm{Ir}$ angles are $96.3^{\circ}\left(100.2^{\circ}\right)$ for $\mathrm{Li}_{3} \mathrm{Ir}_{3} \mathrm{O}_{8}$ and $98.1^{\circ}\left(103.3^{\circ}\right)$ for $\mathrm{Na}_{3} \mathrm{Ir}_{3} \mathrm{O}_{8}$, respectively. However, the sizable differences between Ir-O1 and Ir-O2 bonds still remain in $\mathrm{Li}_{3} \mathrm{Ir}_{3} \mathrm{O}_{8}$. The $\mathrm{Ir}-\mathrm{O}$ bond lengths are 2.043(1.984) ^ for Ir-O1(O2), respectively [see Fig. 1(c)].

Although the reduced distortion results in a slightly smaller split of $J_{\text {eff }}=1 / 2$ bands and thus larger density of states at $E_{\mathrm{F}}$ as compared with $\mathrm{Na}_{3} \mathrm{Ir}_{3} \mathrm{O}_{8}$ [19], the transport, magnetic properties and the electronic specific heat, together with 
the electronic structure calculations, indicate a semimetallic electronic structure in $\mathrm{Li}_{3} \mathrm{Ir}_{3} \mathrm{O}_{8}$ not significantly different from that of $\mathrm{Na}_{3} \mathrm{Ir}_{3} \mathrm{O}_{8}$. We argue that the robustness of the semimetallic state against the lattice distortion can be understood in terms of the energy scale of the two conflicting factors. The semimetallic state is produced by the competition between molecular orbital formation with a bondingantibonding splitting of $\sim 0.6 \mathrm{eV}$ and the strong spin-orbit coupling $\sim 0.5 \mathrm{eV}$ of Ir resulting in the formation of the $J_{\text {eff }}=$ $1 / 2$ state. The difference of crystal field splitting between the two compounds is at most of the order of a tenth of an eV and not large enough to change the competition of these two conflicting factors on the energy scale of $0.5 \mathrm{eV}$.

\section{CONCLUSION}

The hyperkagome iridate $\mathrm{Li}_{3} \mathrm{Ir}_{3} \mathrm{O}_{8}$ was synthesized by an ion-exchange reaction from $\mathrm{Na}_{4} \mathrm{Ir}_{3} \mathrm{O}_{8}$. The measurements of magnetic susceptibility, specific heat, and Seebeck constant suggest a metallic state, and the electronic structure calculation shows that $\mathrm{Li}_{3} \mathrm{Ir}_{3} \mathrm{O}_{8}$ hosts a spin-orbit induced semimetallic band structure. The agreement of calculated and measured RIXS spectra supports the semimetallic state. Despite the slightly reduced distortion of the Ir-O network, $\mathrm{Li}_{3} \mathrm{Ir}_{3} \mathrm{O}_{8}$ essentially shares the same electronic structure with $\mathrm{Na}_{3} \mathrm{Ir}_{3} \mathrm{O}_{8}$. The spin-orbit induced semimetallic electronic structure seems robust against the small change of structural parameters, which we argue to be the consequence of the large energy scale of bonding-antibonding splitting for the molecular orbitals and spin-orbit coupling as compared with crystal field effect from the change in lattice distortion. In $J_{\text {eff }}=1 / 2$ Mott insulators, the magnetic exchanges of $J_{\text {eff }}=$ $1 / 2$ pseudospins, particularly anisotropic couplings of $90^{\circ}$ Ir-O-Ir bond, have a much smaller energy scale of $10 \mathrm{meV}$ than the two important energy scales in the spin-orbit coupling induced semimetals and are sensitive to the local lattice distortion [29]. Therefore, if the $J_{\text {eff }}=1 / 2$ Mott insulator $\mathrm{Li}_{4} \mathrm{Ir}_{3} \mathrm{O}_{8}$ is obtained by, e.g., electrochemical reaction from $\mathrm{Li}_{3} \mathrm{Ir}_{3} \mathrm{O}_{8}$, a much more drastic change of the ground state from that of $\mathrm{Na}_{4} \mathrm{Ir}_{3} \mathrm{O}_{8}$ might be anticipated, which is worth further exploration.

\section{ACKNOWLEDGMENTS}

We thank S. Prill-Diemer and F. Falkenberg for their experimental support. We also thank Science \& Technology Facilities Council (STFC) for the allocation of beamtime on HRPD at the ISIS Neutron and Muon Source (Proposal No. RB1720365). The synchrotron radiation experiment was performed at the BL11XU of SPring- 8 with the approval of the Japan Synchrotron Radiation Research Institute (JASRI) (Proposal No. 2016B3552). This work was partly supported by the Alexander von Humboldt foundation, the Japan Society for the Promotion of Science (JSPS) KAKENHI (No. JP15H05852, 17H01140), and JSPS Core-to-core program "Solid-state chemistry for transition-metal oxides".
[1] J. G. Rau, E. K. H. Lee, and H. Y. Kee, Annu. Rev. Condens. Matter Phys. 7, 195 (2016).

[2] G. Jackeli and G. Khaliullin, Phys. Rev. Lett. 102, 017205 (2009).

[3] Y. Okamoto, M. Nohara, H. Aruga-Katori, and H. Takagi, Phys. Rev. Lett. 99, 137207 (2007).

[4] R. Dally, T. Hogan, A. Amato, H. Luetkens, C. Baines, J. Rodriguez-Rivera, M. J. Graf, and S. D. Wilson, Phys. Rev. Lett. 113, 247601 (2014).

[5] A. C. Shockley, F. Bert, J.-C. Orain, Y. Okamoto, and P. Mendels, Phys. Rev. Lett. 115, 047201 (2015).

[6] G. Chen and L. Balents, Phys. Rev. B 78, 094403 (2008).

[7] M. R. Norman and T. Micklitz, Phys. Rev. B 81, 024428 (2010).

[8] D. Podolsky and Y. B. Kim, Phys. Rev. B 83, 054401 (2011).

[9] I. Kimchi and A. Vishwanath, Phys. Rev. B 89, 014414 (2014).

[10] R. Shindou, Phys. Rev. B 93, 094419 (2016).

[11] T. Mizoguchi, K. Hwang, E. K.-H. Lee, and Y. B. Kim, Phys. Rev. B 94, 064416 (2016).

[12] T. Takayama, A. Yaresko, A. Matsumoto, J. Nuss, K. Ishii, M. Yoshida, J. Mizuki, and H. Takagi, Sci. Rep. 4, 6818 (2014).

[13] A. C. Hannon, A. S. Gibbs, and H. Takagi, J. Appl. Crystallogr. 51, 854 (2018).

[14] A. C. Larson and R. B. Von Dreele, "General Structure Analysis System (GSAS)," Los Alamos National Laboratory Report LAUR 86-748 (2000).
[15] B. H. Toby, J. Appl. Crystallogr. 34, 210 (2001).

[16] V. Antonov, B. Harmon, and A. Yaresko, Electronic Structure and Magneto-Optical Properties of Solids (Kluwer Academic, Dordrecht, 2004).

[17] A. Kotani and S. Shin, Rev. Mod. Phys. 73, 203 (2001).

[18] L. J. P. Ament, M. van Veenendaal, T. P. Devereaux, J. P. Hill, and J. van den Brink, Rev. Mod. Phys. 83, 705 (2011).

[19] See Supplemental Material at http://link.aps.org/supplemental/ 10.1103/PhysRevMaterials.4.075002 for the characterizations of $\mathrm{Li}_{3} \mathrm{Ir}_{3} \mathrm{O}_{8}$ polycrystalline sample, Rietveld analysis of powder neutron diffraction pattern, comparison of physical properties and electronic structure with those of $\mathrm{Na}_{3} \mathrm{Ir}_{3} \mathrm{O}_{8}$, and detailed discussion for the molecular orbital formation, which include Refs. [20,21].

[20] S. Bette, T. Takayama, V. Duppel, A. Poulain, H. Takagi, and R. E. Dinnebier, Dalton. Trans. 48, 9250 (2019).

[21] J. Fujioka, T. Okawa, A. Yamamoto, and Y. Tokura, Phys. Rev. B 95, 121102(R) (2017).

[22] T. Hayakawa, D. Shimada, and N. Tsuda, J. Phys. Soc. Jpn. 58, 2867 (1989).

[23] R. T. Delves, Rep. Prog. Phys. 28, 249 (1965).

[24] B. C. Passenheim and D. C. McCollum, J. Chem. Phys. 51, 320 (1969).

[25] J. Chaloupka, G. Jackeli, and G. Khaliullin, Phys. Rev. Lett. 110, 097204 (2013). 
[26] K. Ishii, I. Jarrige, M. Yoshida, K. Ikeuchi, J. Mizuki, K. Ohashi, T. Takayama, J. Matsuno, and H. Takagi, Phys. Rev. B 83, 115121 (2011).

[27] H. Gretarsson, J. P. Clancy, X. Liu, J. P. Hill, E. Bozin, Y. Singh, S. Manni, P. Gegenwart, J. Kim, A. H. Said, D. Casa, T. Gog, M. H. Upton, H. S. Kim, J. Yu, V. M. Katukuri, L. Hozoi, J. van den Brink, and Y.-J. Kim, Phys. Rev. Lett. 110, 076402 (2013).
[28] The Shannon's ionic radius for $\mathrm{Li}^{+}, \mathrm{Na}^{+}$and $\mathrm{Ir}^{4+}$ ions with the coordination number of 6 is $0.76,1.02$, and $0.625 \AA$, respectively.

[29] S. M. Winter, Y. Li, H. O. Jeschke, and R. Valenti, Phys. Rev. B 93, 214431 (2016).

[30] K. Momma and F. Izumi, J. Appl. Crystallogr. 44, 1272 (2011). 